Portland State University

PDXScholar

\title{
Surface Plasmons of a Graphene Parallel Plate Waveguide Bounded by Kerr-type Nonlinear Media
}

\author{
H. Hajian
}

University of Tabriz

A. Soltani-Vala

University of Tabriz

M. Kalafi

University of Tabriz

Pui T. Leung

Portand State University, hopl@pdx.edu

Follow this and additional works at: https://pdxscholar.library.pdx.edu/phy_fac

Part of the Physics Commons

Let us know how access to this document benefits you.

\section{Citation Details}

Hajian, H., et al. "Surface plasmons of a graphene parallel plate waveguide bounded by Kerr-type nonlinear media." Journal of Applied Physics 115.8 (2014): 083104.

This Article is brought to you for free and open access. It has been accepted for inclusion in Physics Faculty Publications and Presentations by an authorized administrator of PDXScholar. Please contact us if we can make this document more accessible: pdxscholar@pdx.edu. 


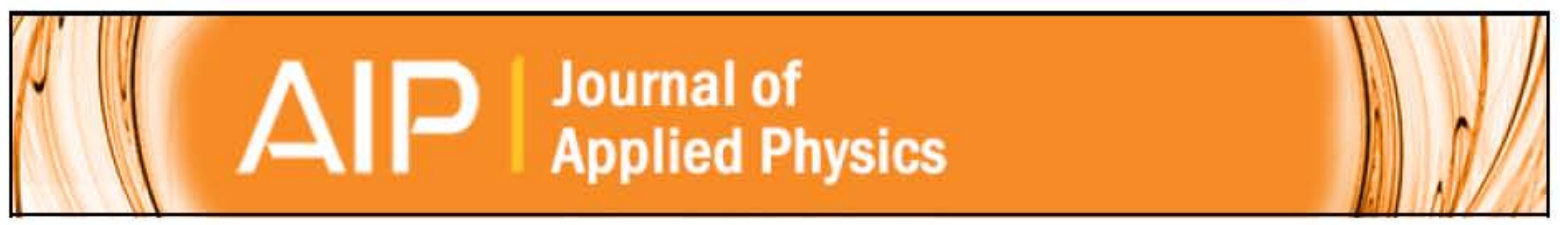

Surface plasmons of a graphene parallel plate waveguide bounded by Kerr-type nonllnear medla

H. Hajian, A. Soltani-Vala, M. Kalafi, and P. T. Leung

Citation: Journal of Applied Physics 115, 083104 (2014); doi: 10.1063/1.4865435

View online: http://dx.doi.org/10.1063/1.4865435

View Table of Contents: http:/scitation.aip.org/content/aip/joumal/jap/115/8?ver=pdfcov

Published by the AIP Publishing

Articles you may be Interested In

Coupled surface plasmon modes of graphene in close proximity to a plasma layer

Appl. Phys. Lett. 103, 201104 (2013); 10.1063/1.4830006

Graphene disk as an ultra compact ring resonator based on edge propagating plasmons

Appl. Phys. Lett. 103, 163114 (2013); 10.1063/1.4826515

Optlm|zing terahertz surface plasmons of a monolayer graphene and a graphene parallel plate wavegulde using one-dimensional photonic crystal

J. Appl. Phys. 114, 033102 (2013); 10.1063/1.4813415

Coupled electromagnetic TE-TM wave propagation in a layer with Kerr nonlinearity

J. Math. Phys. 53, 123530 (2012); 10.1063/1.4769885

Exact surface plasmon dispersion relations in a linear-metal-nonlinear dielectric structure of arbitrary nonlinearity Appl. Phys. Lett. 94, 221102 (2009); 10.1063/1.3144273

AlIP Re-register for Table of Content Alerts Create a profile. 


\title{
Surface plasmons of a graphene parallel plate waveguide bounded by Kerr-type nonlinear media
}

\author{
H. Hajian, ${ }^{1, a)}$ A. Soltani-Vala, ${ }^{1,2}$ M. Kalafi, ${ }^{1,2,3}$ and P. T. Leung ${ }^{4}$ \\ ${ }^{1}$ Department of Solid State Physics, Faculty of Physics, University of Tabriz, Tabriz 51665-163, Iran \\ ${ }^{2}$ Research Institute for Applied Physics and Astronomy, University of Tabriz, Tabriz 51665-163, Iran \\ ${ }^{3}$ Excellent Center for Photonics, University of Tabriz, 51665-163 Tabriz, Iran \\ ${ }^{4}$ Department of Physics, Portland State University, P.O. Box 751, Portland, Oregon 97207-0751, USA
}

(Received 2 December 2013; accepted 22 January 2014; published online 25 February 2014)

\begin{abstract}
The exact dispersion relations of the transverse magnetic surface plasmons (SPs) supported by a graphene parallel plate waveguide (PPWG), surrounded on one or both sides by Kerr-type nonlinear media, are obtained analytically. It is shown that if self-focusing nonlinear materials are chosen as the surrounding media, the SPs localization length (LL) is decreased, while their propagation length (PL) remains unchanged, as compared to those of a typical graphene PPWG. Moreover, PL and LL of the SPs are considerably affected by adjusting nonlinear parts of the dielectric permittivities of the nonlinear media. It is found that using an appropriate defocusing nonlinear material as a substrate of the graphene PPWG, which is surrounded on one side by the nonlinear medium, leads to noticeable enhancement of the propagation and localization characteristics of the surface plasmons. The results presented here can be useful for enhancing capabilities of plasmonic devices based on the graphene PPWG for sensing and waveguide applications. (C) 2014 AIP Publishing LLC. [http://dx.doi.org/10.1063/1.4865435]
\end{abstract}

\section{INTRODUCTION}

Graphene, ${ }^{1-6}$ planar atomic layer of carbon atoms arranged in a honeycomb lattice, has recently attracted a great deal of attention due to its potential applications in optoelectronics and plasmonics. ${ }^{7-12}$ Being tunable [through the tuning of the optical conductivity $\left(\sigma_{g}\right)$ ] by either electrostatic/magnetostatic gating or chemical doping ${ }^{1,2}$ is the unique characteristic of the electronic and optical properties of its doped version. When $\operatorname{Im}\left(\sigma_{g}\right)<0$, weakly guided transverse electric (TE) surface plasmons (SPs) might propagate along a monolayer graphene. ${ }^{13-16}$ Moreover, when $\operatorname{Im}\left(\sigma_{g}\right)>0$, a monolayer graphene effectively behaves as a very thin metal layer capable of supporting only transverse magnetic (TM) SPs. ${ }^{13-15,17-22}$ (The SPs are bound electromagnetic modes propagating at the interface between a dielectric and a conductor, evanescently confined in the perpendicular direction ${ }^{23-25}$ ). The graphene SPs are believed to be a conveniently tunable means for strong light-matter interaction in the IR and terahertz range.,16 Therefore, like previous studies have been done on SPs supported at metalKerr medium interfaces, ${ }^{26-35}$ the nonlinear contribution from substrate to the properties of monolayer graphene SPs has also been studied recently. ${ }^{36}$ It should be noted that, for metal-Kerr medium interfaces with TM electromagnetic waves being supported, the corresponding nonlinear Maxwell equations involve two components of the electric field. By employing some approximations, ${ }^{26-28}$ using semianalytic/analytic approaches ${ }^{29-32,34}$ or numerical methods, ${ }^{33-35}$ the problem has been solved. It was shown in Ref. 36 that the strong electromagnetic field of graphene SPs (the enhancement factor can be as large as $10^{15}$ [Ref. 37])

${ }^{a)}$ Electronic mail: hodjat.hajian@gmail.com. makes the nonlinear contribution from the substrate a considerable factor; i.e., the nonlinear substrate enhances the confinement [decreases the localization length (LL)] of the SPs without impairing their propagation length (PL).

Graphene parallel plate waveguide (PPWG) ${ }^{17}$ in which the separation distance $(D)$ of the graphene sheets is sufficiently large $(\geq 6 \mathrm{~nm}),{ }^{19}$ is also capable of supporting SPs with considerable larger PL, as compared with those of the monolayer graphene. ${ }^{17,19-22}$ In addition to having sensing and waveguide applications, this structure can be a remarkable candidate to design/fabricate miniaturized optical nonlinear couplers ${ }^{38}$ and modulators. ${ }^{39,40}$ The nonlinear contribution from the substrate and cladding to propagation and localization characteristics of the graphene PPWG SPs has not been considered in the previous studies. Since simply using a Kerr-type dielectric permittivity in the typical dispersion relations of the SPs is not justified, in this paper, following Refs. 30-32, we obtain exact dispersion relations for TM SPs of the graphene PPWG, which is bounded either asymmetrically (on one [Fig. 1(a)] or two sides [Fig. 1(b)]) or symmetrically [Fig. 1(c)] by Kerr-type nonlinear media. (It should be noted that the obtained exact dispersion relations can be solved even without detailed knowledge of the field distribution in the nonlinear media). Besides, effect of the nonlinear contributions on the SPs characteristics is also investigated. To the best of our knowledge, the results extracted in this paper have not been previously reported.

\section{MATH AND EQUATIONS}

Fig. 1 depicts side view of the structures considered here. As this figure shows, the graphene plates are placed at $z= \pm D / 2$ and separated by a linear medium of width $\mathrm{D}$ with dielectric constant of $\varepsilon_{2}$. Following Refs. 30-32 and 


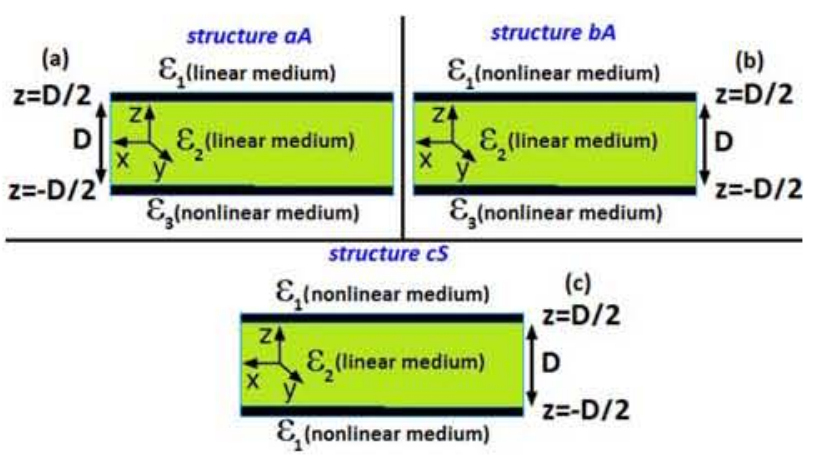

FIG. 1. The panels represent side view of the graphene PPWG, the thick black lines placed at $z= \pm D / 2$ end separated by a linear medium of width $D$ with dielectric constant of $\mathrm{E}_{2}$. In the "structures aA and bA," shown in panels (a) and (b), the graphene PPWG is asymmetrically on one and two sides bounded by the Kerr-type nonlinear media, respectively. Panel (c) shows the "structure $\mathrm{cS}^{\prime \prime}$ in which the graphene PPWG is symmetrically bounded by the Kerr-type nonlinear media. Moreover, " $A$ " ("S") label refers to asymmetric (symmetric) structure, respectively.

starting from the Maxwell's equations for TM waves, the electric field components $E_{x}(z)$ and $E_{z}(z)$ obey

$$
\frac{d E_{x}}{d z}=\frac{-q_{j}^{2} E_{z}}{\beta}, \quad \frac{d\left(\varepsilon_{j} E_{z}\right)}{d z}=-\beta \varepsilon_{j} E_{x}, \quad H_{y}=\frac{-\varepsilon_{0} \varepsilon_{j} \omega E_{z}}{\beta},
$$

where $\mathbf{E}(\mathbf{r}, t)=\frac{1}{2}\left\{i E_{x} \hat{x}+E_{z} \hat{z}\right\} \exp [i(\omega t-\beta x)]+$ c.c. (the two electric field components are $\pi / 2$ out of phase), $q_{j}^{2}=\beta^{2}-\varepsilon_{j} k_{0}^{2}, k_{0}=\omega / c$ is the free space wave vector, and $\beta$ is the SP wave number. Besides, $\varepsilon_{j}(j=1,2,3)$ is the dielectric permittivity of the layers and it is, for the isotropic nonlinearity considered here, assumed to be of the Kerr-type (i.e., $\varepsilon_{j}=\varepsilon_{j}^{n t}=\varepsilon_{j}^{l}+\alpha_{j}|\mathbf{E}|^{2}(\mathrm{j}=3$ [for the "structure aA"], $\mathrm{j}=1,3$

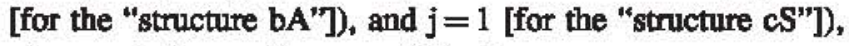
where $\alpha_{j}$ is the nonlinear coefficient.

First, we consider the asymmetric graphene PPWG structure ["structure $\left.\mathrm{aA}^{\prime \prime}\right]$ for which only the substrate is a nonlinear medium $\left(\varepsilon_{3}^{n I}=\varepsilon_{3}^{b}+\alpha_{3}|\mathbf{E}|^{2}\right)$ and the cladding is a linear one $\left(\varepsilon_{1}=\varepsilon_{1}\right)$. Considering $E_{x}(z)$ for the linear regions as

$$
E_{x}(z)=\left\{\begin{array}{ll}
E_{\frac{D}{2} x} \exp \left(-q_{1}[z-D / 2]\right) & z \geq D / 2 \\
A_{1} \exp \left(q_{2} z\right)+B_{1} \exp \left(-q_{2} z\right) & -D / 2 \leq z<D / 2
\end{array}\right\},
$$

where $\quad E_{q_{x}}=E_{x}(z=D / 2), \quad A_{1}=\frac{e-q^{D / 2}}{2}\left[1+q_{2} \Lambda_{1} / e_{2}\right] E_{p_{x}}$, $B_{1}=\frac{e_{2} D / 2}{2}\left[1-q_{2} \Lambda_{1} / \varepsilon_{2}\right] E_{q_{x}}$, and $\Lambda_{1}=\sigma_{g}^{l p p} /\left(i \epsilon_{0} \omega\right)-\varepsilon_{1}^{l} / q_{1}$. Note that the boundary conditions for TM polarization at $z=$ D/2 [Refs. 41-43]

$$
\begin{aligned}
& \left.\frac{\varepsilon_{\frac{p^{+}}{2}}}{\left(i q_{\frac{p^{+}}{2}}\right)^{2}} \frac{d E_{x}}{d z}\right|_{z=\frac{p^{+}}{2}}-\left.\frac{\frac{\sigma_{0}}{2}}{\left(i q_{p^{-}}\right)^{2}} \frac{d E_{x}}{d z}\right|_{z=\frac{\sigma^{-}}{2}}=\left.\frac{\sigma_{g}(\omega)}{i \epsilon_{0} \omega} E_{x}\right|_{z=\frac{0^{2}}{2}}, \\
& \left.E_{x}\right|_{z=\frac{p^{+}}{2}}=\left.E_{x}\right|_{z=\frac{0^{-}}{2}},
\end{aligned}
$$

have already been fulfilled. Moreover, in this paper, the optical conductivity of the graphene plates, for frequencies $\omega \gg\left(k \nu_{F}, \tau^{-1}\right)$, at temperature $T$, is chosen as $\sigma_{g}=\sigma_{g}^{i n t a}$ $+\sigma_{8}^{\text {inter }}$, where ${ }^{41,42}$

$$
\begin{aligned}
\sigma_{g}^{i n f r a} & =\frac{e^{2}}{4 \hbar} \frac{i}{2 \pi}\left\{\frac{16 k_{B} T}{\hbar \Omega} \ln \left(2 \cosh \left(\frac{\mu}{2 k_{B} T}\right)\right)\right\}, \\
& =\frac{e^{2}}{4 \hbar} \frac{i}{2 \pi} \frac{8 \mu}{\hbar \Omega}\left(\mu \gg k_{B} T\right),
\end{aligned}
$$

and

$$
\begin{aligned}
\sigma_{g}^{\text {inter }}= & \frac{e^{2}}{4 \hbar}\left\{0.5+\frac{1}{\pi} \arctan \left(\frac{\hbar \Omega-2 \mu}{2 k_{B} T}\right)\right. \\
& \left.-\frac{i}{2 \pi} \ln \frac{(\hbar \Omega+2 \mu)^{2}}{(\hbar \Omega-2 \mu)^{2}+\left(2 k_{B} T\right)^{2}}\right\} .
\end{aligned}
$$

Here, $\Omega=\omega+i \tau^{-1}, e$ is the charge of an electron, $\tau$ is the electron relaxation time, $k$ is the wave vector, $k_{F}$ is the Fermi velocity of electrons in graphene, $k_{B}$ is the Boltzmann constant, and $\mu$ is the chemical potential determined by the electron concentration, which can be controlled by gating. Applying the boundary conditions at $z=-D / 2$ yields

$$
\begin{aligned}
\left.\frac{d E_{x}}{d z}\right|_{s=\frac{p}{2}^{-}} & =\frac{q_{3,-\frac{p^{-}}{2}}^{2}}{e_{3,-\frac{p^{-}}{2}}^{n d}} \Delta_{2} E_{-\frac{p^{2}}{2},} \\
& =-\frac{q_{3,-\frac{p^{-}}{2}}^{2}}{\beta} E_{\frac{p^{-}}{2},}
\end{aligned}
$$

and the last line follows from Eq. (1), $E_{-\frac{p}{2}}=E_{z}\left(z=-\frac{D^{-}}{2}\right)$, $E_{-\frac{p}{2} x}=E_{x}(z=-D / 2), q_{3,-\frac{p^{-}}{2}}=\left.q_{3}\right|_{z=-\frac{p^{-}}{2}}, \varepsilon_{3, \frac{p^{-}}{2}}^{n n l}=\left.\varepsilon_{3}^{n l}\right|_{z=-\frac{p^{-}}{2}}$ $=\vec{z}_{3}+\alpha_{3} E_{-\frac{2}{2}}^{2}, \quad$ and $\quad \Delta_{2}=\sigma_{g}^{d o w n} /\left(i \epsilon_{0} \omega\right)+\frac{\sigma_{2}}{q_{2}}$

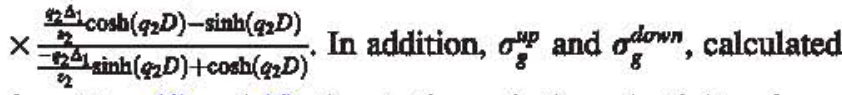
from Eqs. (4) and (5), denote the optical conductivity of graphene sheets placed at $z=D / 2$ and $z=-D / 2$, respectively, and could be tuned through their respective chemical potential $\mu_{g}^{u p}$ and $\mu_{g}^{d o w n}$. Equation ( 6 ) yields simply

$$
E_{-\frac{p}{2} x}=-\frac{\varepsilon_{3,-\frac{p-}{2}}^{n l}}{\beta \Delta_{2}} E_{-\frac{p^{-}}{2}} \text {. }
$$

Using Eqs. (1) and (7) and the first integral obtained in Eq. (6) of Ref. 30 , one can obtain another relation between $E_{-\frac{1}{2} x}$ and $E_{-\frac{a^{-}}{2} z}$ as follows

$$
\begin{aligned}
& \frac{\alpha_{3}}{2} E_{-\frac{p}{2} x}^{4}+\left(k_{0}^{2} \Delta_{2}^{2}+\varepsilon_{3}^{l}\right) E_{-\frac{p}{2} x}^{2} \\
& \quad+\Delta_{2} \beta E_{\frac{D}{2} x^{\frac{D}{2}} z} E_{\frac{p^{-}}{2}}-\frac{\alpha_{3}}{2} E_{\frac{p^{-}}{2} z}^{4}=0 .
\end{aligned}
$$

Furthermore, using Eq. (7) together with the relation $E_{\frac{n}{2}}^{2}=$ $E_{-\frac{p^{2} x}{2}}^{2}+E_{-\frac{p^{-}}{2} z}^{2}$ results in 


$$
\begin{aligned}
E_{-\frac{D^{2}}{2}}^{2} & =X_{1}, \quad E_{-\frac{D^{-}}{2} z}^{2}=\left(\frac{\beta \Delta_{2}}{\varepsilon_{3,-\frac{p^{-}}{2}}^{n l}}\right)^{2} X_{1}, \\
E_{\frac{D}{2} x} E_{\frac{D^{-}}{2} z} & =-\left(\frac{\beta \Delta_{2}}{\varepsilon_{3,-\frac{D^{-}}{2}}^{n l}}\right) X_{1},
\end{aligned}
$$

where $X_{1}=E_{\frac{D}{2}}^{2} /\left[1+\left(\beta \Delta_{2} / \varepsilon_{3,-\frac{D}{2}}^{n l}\right)^{2}\right]$. Combining Eqs. (8) and (9), the exact dispersion relation for the SPs supported by the "structure aA," named as "aA SPs," is finally obtained

$$
\frac{\sqrt{\varepsilon_{3, \frac{p^{-}}{2}}^{n l}\left(\frac{\alpha_{3}}{2} E_{-\frac{p}{2}}^{2}+\varepsilon_{3}^{l}\right)}}{q_{3,-\frac{p^{-}}{2}}}=\Delta_{2} \sqrt{1+\frac{\alpha_{3} E_{\frac{n}{2}}^{2} \beta^{2}}{2 \varepsilon_{3,-\frac{p^{-}}{2}}^{n l} \times q_{3,-\frac{p^{-}}{2}}^{2}}},
$$

Letting $\alpha_{3} \rightarrow 0$, Eq. (10) reduces to the well-known dispersion relation of TM SPs (named as "A SPs") of the typical asymmetric graphene $\mathrm{PPWG}^{17,19-22}$

$$
\tanh \left(q_{2} D\right)=-\frac{\Gamma_{1}+\Gamma_{3}}{1+\Gamma_{1} \Gamma_{3}}(A S P s),
$$

where $\Gamma_{1}=\frac{q_{2}}{\varepsilon_{2}}\left[\varepsilon_{1}^{l} / q_{1}^{l}+i \sigma_{g}^{u p} /\left(\epsilon_{0} \omega\right)\right]$ and $\Gamma_{3}=\frac{q_{2}}{\varepsilon_{2}}\left[\varepsilon_{3}^{l} / q_{3}^{l}\right.$ $\left.+i \sigma_{g}^{d o w n} /\left(\epsilon_{0} \omega\right)\right]^{2}$.

Next, we consider the second asymmetric graphene PPWG structure in which both the substrate $\left(\varepsilon_{3}^{n l}\right)$ and cladding $\left(\varepsilon_{1}^{n l}=\varepsilon_{1}^{l}+\alpha_{1}|\mathbf{E}|^{2}\right)$ are nonlinear media ["structure bA," shown in Fig. 1(b)]. In this case, the solution for $E_{x}(z)$ in the linear region can be considered as

$$
E_{x}(z)=A_{2} \exp \left(q_{2} z\right)+B_{2} \exp \left(-q_{2} z\right), \quad-D / 2 \leq z \leq D / 2
$$

with $A_{2}=\frac{E_{\frac{R_{x}}{2}}-e_{q_{2} D} E_{D_{x}}}{e^{-q_{2} D / 2}-e^{3 q_{2} D / 2}}, \quad B_{2}=\frac{E_{D_{x}}-e^{q_{2} D} E_{R_{x}}}{e^{-q_{2} D / 2}-e^{3 q_{2} D / 2}}$. Applying the boundary conditions at $z=+D / 2$, we have

$$
\begin{aligned}
\left.\frac{d E_{x}}{d z}\right|_{z=\frac{\underline{B}^{+}}{2}} & =-\frac{q_{1, \frac{D^{+}}{2}}^{2}}{\varepsilon_{1, \frac{D^{+}}{2}}^{n}} \Delta_{4 a} E_{\frac{D}{2}} x \\
& =-\frac{q_{1, \frac{D^{+}}{2}}^{2}}{\beta} E_{\frac{D^{+}}{2} z^{+}},
\end{aligned}
$$

where the last line follows from Eq. (1), $E_{\frac{D^{+}}{2}}=E_{z}\left(z=\frac{D}{2}^{+}\right)$, $q_{1, \frac{D^{+}}{2}}=\left.q_{1}\right|_{z=\frac{D^{+}}{2}}, \varepsilon_{1, \frac{D^{+}}{2}}^{n l}=\left.\varepsilon_{1}^{n l}\right|_{z=\frac{D^{+}}{2}}=\varepsilon_{1}^{l}+\alpha_{1} E_{\frac{D}{2}}^{2}, \Delta_{4 a}=\sigma_{g}^{u p} /\left(i \epsilon_{0} \omega\right)$ $-\varepsilon_{2} \Delta_{3 a} / q_{2}, \Delta_{3 a}=\frac{2 e^{q_{2} D / 2} M-C_{1}}{C_{2}}, C_{1}=e^{-q_{2} D / 2}+e^{3 q_{2} D / 2}, C_{2}=$ $e^{-q_{2} D / 2}-e^{3 q_{2} D / 2}$, and $M=E_{-\frac{D}{2} x} / E_{\frac{D}{2} x}$. Equation (13) yields

$$
E_{\frac{D}{2} x}=+\frac{\varepsilon_{1 \frac{D^{+}}{2}}^{n l}}{\beta \Delta_{4 a}} E_{\frac{D^{+}}{2} z^{\prime}} \text {. }
$$

Using Eqs. (1) and (14), and the first integral, ${ }^{30}$ in this case, we have another relation between $E_{\frac{D}{2} x}$ and $E_{\frac{D}{2}{ }_{z}}$ as follows

$$
\frac{\alpha_{1}}{2} E_{\frac{D}{2} x}^{4}+\left(k_{0}^{2} \Delta_{4 a}^{2}+\varepsilon_{1}^{l}\right) E_{\frac{D_{2} x}{2}}^{2}-\Delta_{4 a} \beta E_{\frac{p_{2} x}{2} E_{\frac{D^{+}}{2} z}}-\frac{\alpha_{1}}{2} E_{\frac{p^{+} z}{2}}^{4}=0 .
$$

Besides, using Eq. (14) together with the relation $E_{\frac{D}{2}}^{2}=E_{\frac{D}{2} x}^{2}$ $+E_{\frac{D^{+}}{2}}^{2}$, the following relations can be obtained

$$
\begin{aligned}
E_{\frac{D^{2}}{2}}^{2} & =X_{2}, \quad E_{\frac{D^{+}}{2} z}^{2}=\left(\frac{\beta \Delta_{4 a}}{\varepsilon_{1, \frac{D^{+}}{2}}^{n l}}\right)^{2} X_{2}, \\
E_{\frac{D}{2} x^{\frac{D}{2}}{ }^{+} z} & =+\left(\frac{\beta \Delta_{4 a}}{\varepsilon_{1, \frac{D^{+}}{2}}^{n l}}\right) X_{2},
\end{aligned}
$$

where $X_{2}=E_{\frac{0}{2}}^{2} /\left[1+\left(\beta \Delta_{4 a} / \varepsilon_{1, \frac{D^{+}}{2}}^{n l}\right)^{2}\right]$. Combining Eqs. (15) and (16), we arrive at

$$
\frac{D_{a 1}}{q_{1, \frac{D^{+}}{2}}}=\Delta_{4 a} D_{a 2}
$$

where $\quad D_{a 1}=\sqrt{\left.\varepsilon_{1, \frac{p^{2}}{2}}^{n l} \frac{\alpha_{1}}{2} E_{\frac{D}{2}}^{2}+\varepsilon_{1}^{l}\right)} \quad$ and $\quad D_{a 2}$ $=\sqrt{1+\alpha_{1} E_{\frac{D}{2}}^{2} \beta^{2} /\left(2 \varepsilon_{1, \frac{D^{+}}{2}}^{n l} \times q_{1, \frac{p^{+}}{2}}^{2}\right)}$. In addition, applying the boundary conditions at $z=-D / 2$ and following similar procedures for arriving at Eq. (10), the below relation is obtained

$$
\frac{D_{b 1}}{q_{3,-\frac{D^{-}}{2}}}=\Delta_{4 b} D_{b 2}
$$

where

$$
D_{b 1}=\sqrt{\varepsilon_{3,-\frac{D^{2}}{2}}^{n l}\left(\frac{\alpha_{3}}{2} E_{-\frac{D}{2}}^{2}+\xi_{3}^{J}\right)},
$$

$=\sqrt{1+\alpha_{3} E_{-\frac{p}{2}}^{2} \beta^{2} /\left(2 \varepsilon_{3,-\frac{p^{-}}{2}}^{n l} \times q_{3, \frac{p^{-}}{2}}^{2}\right)}, \quad \Delta_{4 b}=\sigma_{g}^{d o w n} /\left(i \epsilon_{0} \omega\right)$ $+\varepsilon_{2} \Delta_{3 b} / q_{2}$, and $\Delta_{3 b}=\frac{-2 e^{a_{2} D / 2} M^{-1}+C_{1}}{C_{2}}$. Finally, eliminating $M$ between Eqs. (17) and (18), the exact dispersion relation for "bA SPs," supported by the "structure bA," is obtained

$$
F_{a} F_{b}=-4 \exp \left(q_{2} D\right) \quad(b A S P s) .
$$

Here, $F_{a}=\frac{-\sigma_{2} E_{a} C_{2}}{\varepsilon_{2}}+C_{1}, E_{a}=D_{a 1} /\left(D_{a 2} q_{1, \frac{p^{+}}{}}\right)+i \sigma_{g}^{\mu p} /\left(\epsilon_{0} \omega\right)$, $F_{b}=\frac{q_{2} E_{b} C_{2}}{\varepsilon_{2}}-{\stackrel{C}{\varepsilon_{1}}}_{1}$, and $E_{b}=D_{b 1} /\left(D_{b 2} q_{3,-\frac{D^{-}}{2}}\right)+i \sigma_{g}^{d o w n} /\left(\epsilon_{0} \omega\right)$. It is worth noting that letting $\left(\alpha_{1}, \alpha_{3}\right) \rightarrow 0$, the above equation also reduces to Eq. (11).

Finally, we consider the symmetric graphene PPWG structure in which both the substrate and cladding are the same nonlinear medium $\left(\varepsilon_{1}^{n l}\right)$ ["structure $c S$ "]. In this case, the odd and even solutions for $E_{x}(z)$ in the linear region are

$$
\begin{aligned}
E_{x}(z)= & \left\{\begin{array}{ll}
E_{+\frac{D}{2} x} \sinh \left(q_{2} z\right) / \sinh \left(q_{2} D / 2\right) & (\text { odd }) \\
E_{+\frac{D_{2}}{2}} \cosh \left(q_{2} z\right) / \cosh \left(q_{2} D / 2\right) & \text { (even) }
\end{array}\right\} \\
& -D / 2 \leq z \leq D / 2
\end{aligned}
$$

Applying the boundary conditions at $z=D / 2$ and following similar procedures leading to Eq. (17), we arrive at the exact odd dispersion relation for "cS SPs" of the "cS structure"

$$
\begin{aligned}
& \frac{\sqrt{\varepsilon_{1, \frac{D^{+}}{2}}^{n l}\left(\frac{\alpha_{1}}{2} E_{\frac{p}{2}}^{2}+\varepsilon_{1}^{l}\right)}}{q_{1, \frac{p^{+}}{2}}}= \Delta_{5} \sqrt{1+\frac{\alpha_{1} E_{\frac{D}{2}}^{2} \beta^{2}}{2 \varepsilon_{1, \frac{p^{+}}{2}}^{n l} \times q_{1, \frac{p^{+}}{2}}^{2}}}, \\
&\left(c S S P s, E_{x} \text { odd }\right),
\end{aligned}
$$


where $\Delta_{5}=\sigma_{g}^{u p} /\left(i \epsilon_{0} \omega\right)-\varepsilon_{2} \operatorname{coth}\left(q_{2} D / 2\right) / q_{2}$ and $\sigma_{g}^{u p}=\sigma_{g}^{d o w n}$. Besides, substituting $\Delta_{6}$ for $\Delta_{5}$ in Eq. (21), the exact even dispersion relation of "cS SPs" can be obtained $\left[\Delta_{6}=\sigma_{g}^{\mu p} /\left(i \epsilon_{0} \omega\right)-\varepsilon_{2} \tanh \left(q_{2} D / 2\right) / q_{2}\right]$. Furthermore, when $\alpha_{1} \rightarrow 0$, we reduce back to the well-known dispersion relations of TM SPs of the typical symmetric graphene PPWG $^{19,22}$

$$
\operatorname{coth}\left(q_{2} D / 2\right)=\left\{\begin{array}{cc}
-\Gamma_{1} & \left(E_{x} \text { odd }\right) \\
-\frac{1}{\Gamma_{1}} & \left(E_{x} \text { even }\right)
\end{array}\right\}(\text { SSPs }) .
$$

It is worth noting that letting $\sigma_{g}^{\text {up down }} \rightarrow 0$ in Eqs. (10), (19), and (21), the exact dispersion relations of TM waves (SPs) guided by a thin dielectric film (thin metal film) bounded by nonlinear media can also be obtained. Note that in the above formulation, we have not taken into account the nonlinear optical response of the graphene layer, which can be significant (see, e.g., Refs. 44 and 45). We have limited ourselves to the same restrictions as applied in several previous works [Refs. 17 and 19-22], which had considered only the linear graphene response in the form " $\sigma_{g}^{l i n} \mathbf{E}$ " for the surface currents. This will be justified in our following numerical studies as long as the optical frequency is not too low (e.g., $f$ to be above $\sim 1 \mathrm{THz}$ ) for a certain given field strength of the wave. For example, we have performed a comparative study of the nonlinear optical conductivity for graphene given in Ref. 38 with the nonlinear permittivity for the Kerr medium used in our present study in Sec. III assuming the later is largely non-dispersive within the frequency range of our study. ${ }^{30-32}$ For a given field strength of $\sim 10^{6} \mathrm{~V} / \mathrm{m}$, we have found that the graphene nonlinear response is insignificant compared to that of the Kerr medium for frequencies above $\sim 0.6 \mathrm{THz}$.

\section{RESULTS AND DISCUSSION}

To investigate effect of the substrate and cladding nonlinearity on the SPs supported by the graphene PPWG, we focus on the following parameters: $\alpha_{3}=6.4 \times 10^{-12} \mathrm{~m}^{2} / \mathrm{V}^{2},{ }^{30-32}$ unless otherwise stated, $\varepsilon_{3}^{l}=7.84$ (for aA structure). ${ }^{33,36}$ For "bA structure," $\alpha_{1}=3 \times 6.4 \times 10^{-12} \mathrm{~m}^{2} / \mathrm{V}^{2}$, unless otherwise stated, $\varepsilon_{1}^{l}=2.25$ and, for the symmetric cS structure, $\varepsilon_{1}^{n l}=\varepsilon_{3}^{n l}$. Dielectric constant $\left(\varepsilon_{2}\right)$ and width $(D)$ of the graphene PPWG core medium are also taken to be 4 and $10 \mathrm{~nm}^{21}$ Moreover, $\tau=1.35 \times 10^{-13} \mathrm{~s},{ }^{18} T=300 \mathrm{~K}, \mu^{u p}=0.2 \mathrm{eV}, \mu^{\text {down }}=0.3 \mathrm{eV}$ (for " $\mathrm{aA}$ and bA structures"), $\mu^{u p}=\mu^{d o w n}=0.2 \mathrm{eV}$ (for "cS Structure") are used for computing the optical conductivity of graphene. We also assume an electric field intensity of $E_{ \pm D^{ \pm} / 2}^{2}=0.683 \times 10^{12} \mathrm{~V}^{2} / \mathrm{m}^{2}$ [Ref. 32] at the interfaces of graphene and the nonlinear media. Moreover, it has to be noted that for results presented here, $2 \pi / \operatorname{Re}(\beta), 1 / 2 \operatorname{Im}(\beta)$, and $1 / \operatorname{Re}\left(q_{j}\right)$ are the wave length $\left(\lambda_{s P}\right), P L$, and the $L L$ of the SPs, respectively. Therefore, we examine the localization and propagation characteristics of the SPs by considering the ratios $L L / D$ and $P L / \lambda_{S P}$. The $P L / \lambda_{S P}$ indicates losses of the SPs and is an estimate of how many wavelengths can a SP propagate before it loses most of its energy. Since the SPs of a graphene PPWG are highly propagating in terahertz frequencies, ${ }^{17-19,21,22}$ the numerical results of this paper are presented in this range. Besides, we also focus only on highly localized SPs, i.e., SPs with $L L / D<100$. Consequently, the calculations are done in frequencies lower than the optical phonon loss threshold, $f_{O p h} \approx 0.2 \mathrm{eV}$ [Ref. 18], and the effects of absorption losses due to the optical phonons are neglected. Moreover, it is worth noting that in investigating SPs in a lossy medium, the wave number is complex $\left(\beta=\beta^{\prime}+i \beta^{\prime \prime}\right)$ and as a result $q_{j}=q_{j}^{\prime}+i q_{j}^{\prime \prime}$. In the case of graphene and graphene PPWG, $\beta^{\prime \prime}$ is small and consequently $q_{j} \approx q_{j}^{\prime}$. Therefore, the other point should be noticed here is that by applying the condition $q_{j}^{\prime}>0$ in calculating the SPs, only the bound modes are considered. ${ }^{19}$

Fig. 2 represents the normalized propagation and localization length of the SPs of the aA and bA [panels (a) and (b)] and cS structures [panels (c) and (d)] with and without nonlinear contribution from the substrate and cladding. It is clear from panels (a) and (c) that the presence of the nonlinear substrate and cladding has no considerable effect on the propagation length of the SPs (actually there is almost no difference between the $P L$ of SPs with and without nonlinear media). However, as clearly shown in panels (b) and (d), their nonlinear contribution noticeably improves the SPs localization of the upper branch. To obtain a concise insight into the mentioned decrease in the $L L$ of the upper branch SPs, we focus on the SPs at a typical $f=6 \mathrm{THz}$ frequency. At this frequency, the normalized localization length (propagation length) of A, aA, and bA Sps are 41.92 (0.4043), 39.53 (0.4043), and $27.36(0.3995)$, respectively. Moreover, those of S and cS SPs are, respectively, 23.25 (0.3931) and $19.42(0.3927)$. Therefore, for the considered structures here, we can conclude that (i) the SPs of the symmetric structure with and without the nonlinear contribution are noticeably more localized (insignificantly less propagating) than those of the asymmetric ones; (ii) for both symmetric and asymmetric structures, the presence of self-focusing nonlinear materials $(\alpha>0)$ as the substrate and the cladding leads to a decrease in localization length of the upper branch SPs for $f>4 \mathrm{THz}$ [see upper branches in panels (b) and (d)].

The nonlinear part in the dielectric permittivity of the substrate and cladding media depends on both light intensity and the nonlinear coefficient. Therefore, considering the effect of changes in $\alpha_{j} E_{ \pm D / 2}^{2}$ on the propagation and localization characteristics of the SPs is also an important issue. Fig. 3 shows the dependence of $P L$ and $L L$ of the $\mathrm{aA}, \mathrm{bA}$, and $\mathrm{cS}$ SPs on changes in the nonlinear part of the dielectric permittivity, at $f=6 \mathrm{THz}$ (the typical above-mentioned frequency). It is obvious from this figure that changes in $\alpha_{j} E_{ \pm D / 2}^{2}$ significantly affects the SPs propagation and localization length. And, for specific values of $\alpha_{j} E_{ \pm D / 2}^{2}$, SPs with considerable larger (smaller) propagation length (localization length), defined as optimized SPs, can be supported by the considered structures [note that the specific values, shown with red arrows, are $\alpha_{3} E_{-D / 2}^{2}=-5.221$ in panel (a), $\alpha_{1} E_{+D / 2}^{2}=-1.497$ in panel (b), $\alpha_{3} E_{-D / 2}^{2}=-5.216$ in panel (c), and $\alpha_{1} E_{+D / 2}^{2}=-5.171$ in panel (d)], respectively. Therefore, by comparing these panels, we can conclude that when $\alpha_{3} E_{-D / 2}^{2}=-5.221$, the "aA structure" is capable of supporting the optimized SP [for the optimized SP, $P L / \lambda_{S P}=0.5551$, and $L L / D=8.833$. The normalized PL and LL of this mode have simultaneously largest 

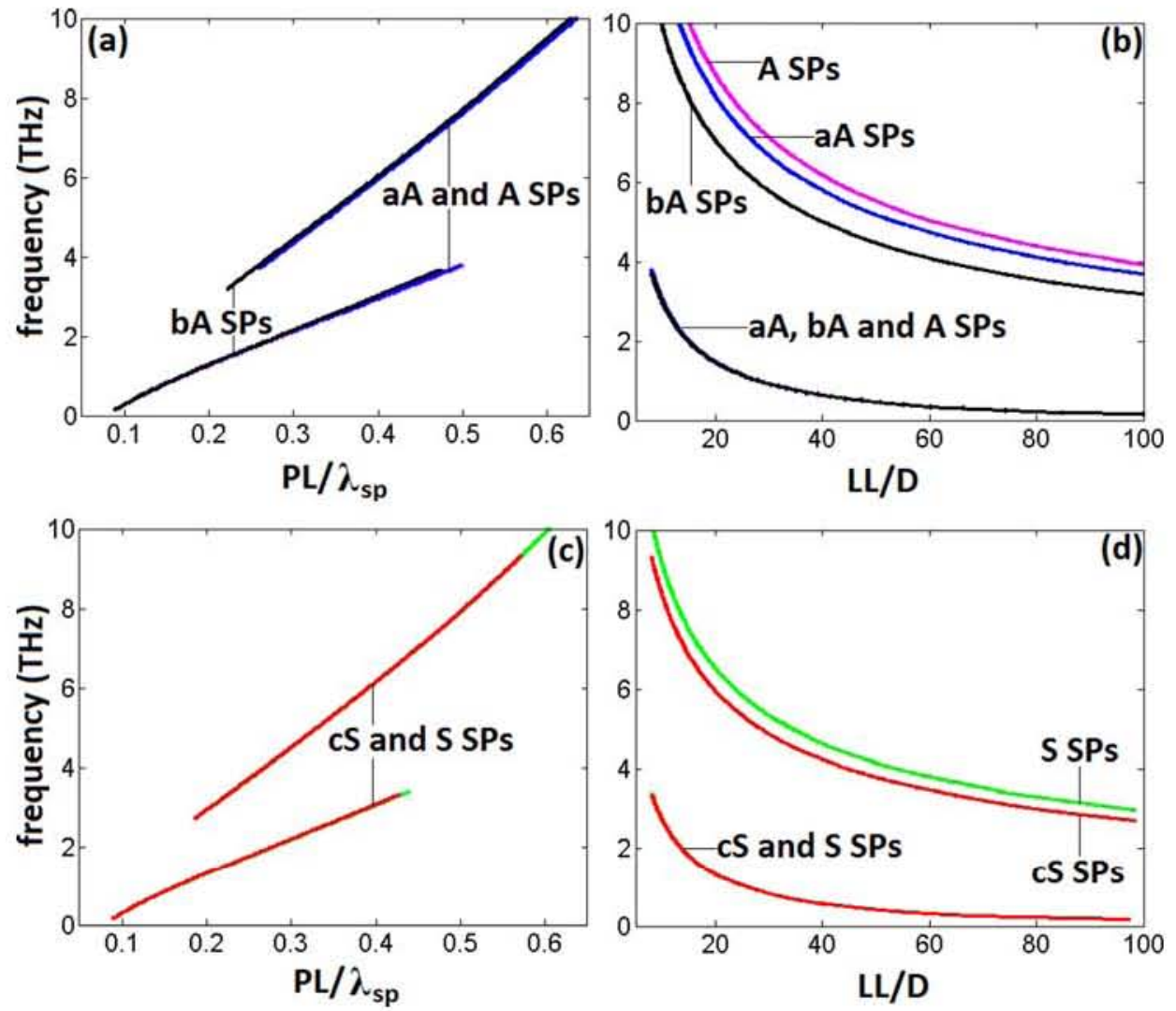

FIG. 2. Panels (a) and (b) depict normalized propagation $\left(P L / \lambda_{S P}\right)$ and localization $(L L D)$ length of $\mathrm{A}\left(a_{j} \rightarrow 0\right)$, aA and bA SPs, respectively. Panels $(c)$ and (d) represent those of $S\left(\alpha_{1} \rightarrow 0\right)$ and $c S S P$.
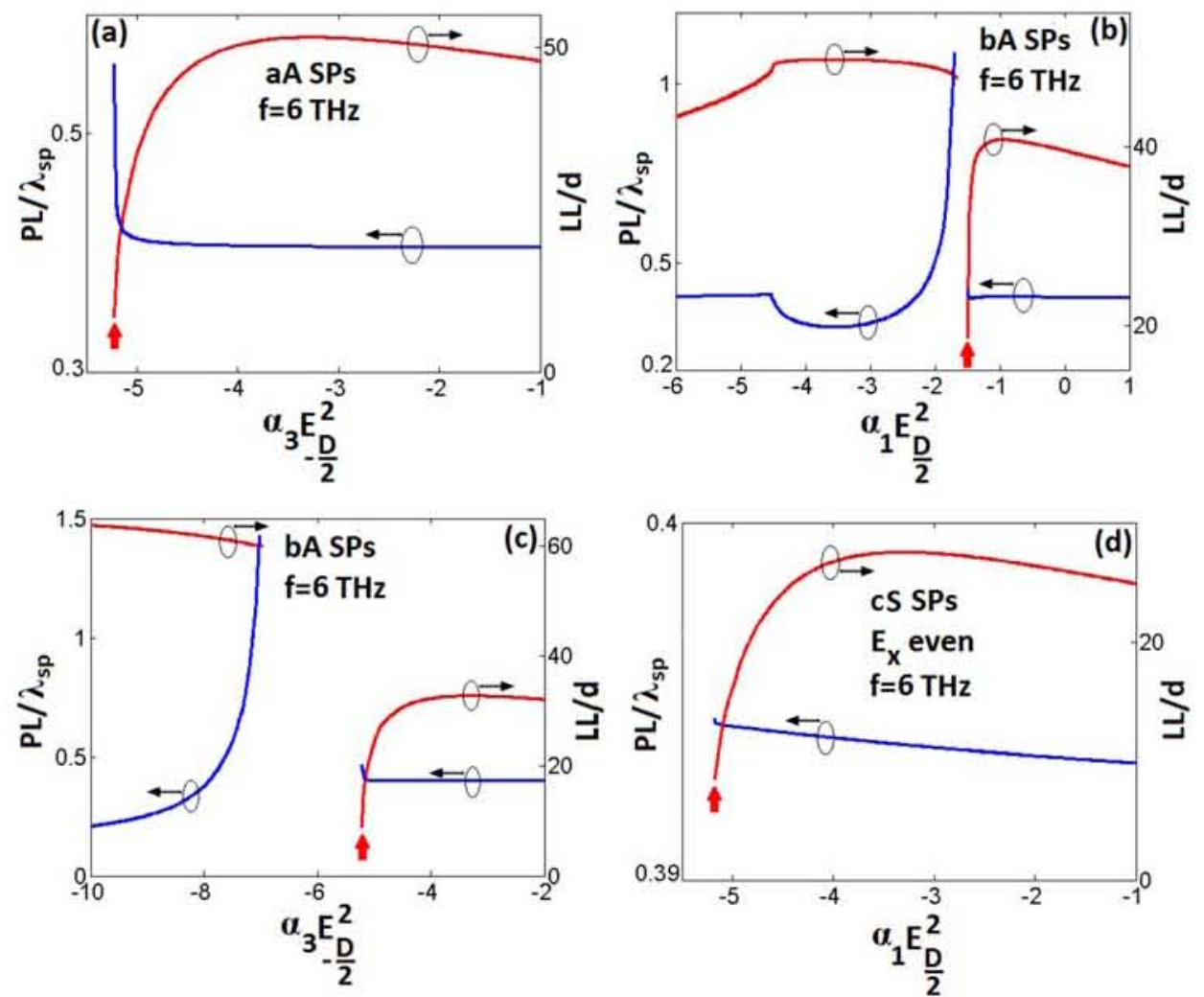

FIG. 3. Dependence of nomalized propagation and localization length of aA [panel (a)], bA [panels (b) and (c)], and cS [panel (d)] SPs on changes in $\alpha_{j} E_{ \pm D^{ \pm} / 2}^{2}$ at $f=6 \mathrm{THz}$. 
and smallest values, respectively, among those shown in Figs. 2 and 3 at $f=6 \mathrm{THz}$ ]. To obtain insight into the problem, Fig. 4 is plotted. This figure shows propagation and localization characteristics of aA SPs when $\alpha_{3} E_{-D / 2}^{2}=-5.221$. Note that to make a better comparison, aA, bA, and cS SPs depicted in Fig. 2 (when $\alpha_{1,3} E_{ \pm D / 2}^{2}=8.991,2.997$ ), are also shown in this figwe. It is clear from panel (a)[(b)] of Fig. 4 that when $\alpha_{3} E_{-D / 2}^{2}=-5.221$, aA SPs with considerably larger (smaller) propagation length (localization length), for $f>4 \mathrm{THz}$, can be supported by the graphene PPWG [e.g., in this case, at $f=6 \mathrm{THz}$, the propagation (localization) length of the optimized SP is increased (decreased) by $41.2 \%$ (62\%), as compared with those of the typical S SP]. As a result, by using an appropriate defocusing nonlinear material $(\alpha<0)$ as a substrate in the "aA structure," it is possible to improve propagation and localization characteristics of the SPs supported by a graphene PPWG. We also investigate terahertz bA SPs when $\alpha_{1} E_{+D / 2}^{2}=$ -1.149 and/or $\alpha_{3} E_{-D / 2}^{2}=-5.216$ [red arrows in Figs. 3(b) and $3(\mathrm{c})$, respectively] and $\mathrm{cS} S P s$ when $\alpha_{1} E_{ \pm D / 2}^{2}=-5.171$ [red arrow in Fig. 3(d)]. We shall skip the details and only mention the result. It is found that although the nonlinear
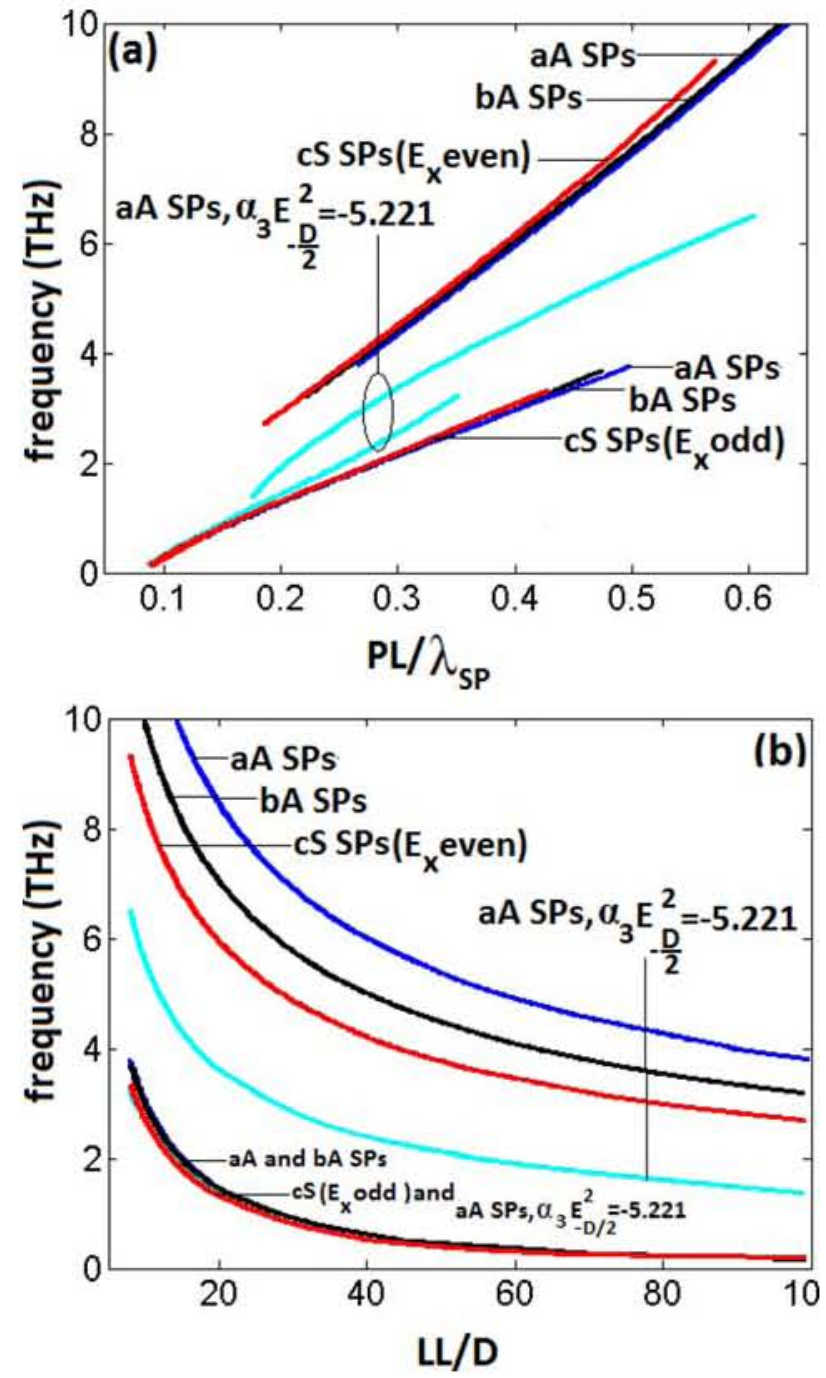

FIG. 4. (a) and (b) show nomalized propagation and localization length of aA SPs when $\alpha_{3} E_{-D-/ 2}^{2}=-5.221$. For better comparison aA, bA, and cS modes depicted in Fig. 2 are also presented here. contribution of the substrate and cladding with a negative nonlinear coefficient always increase the localization of the SPs of the structures shown in Fig. 1, the optimized SPs can only be supported by the "aA structure." Consequently, in contrast to a single layer graphene on a nonlinear substrate, ${ }^{36}$ by choosing an appropriate defocusing material as a substrate, for $f>4 \mathrm{THz}$, it is possible to enhance both propagation and localization of the terahertz surface plasmons of a graphene PPWG, which is asymmetric on one side bounded by nonlinear media.

\section{CONCLUSION}

In conclusion, we have obtained exact dispersion relations for the TM surface plasmons of a graphene parallel plate waveguide, which is either asymmetrically (on one or both sides) or symmetrically bounded by Kerr-type nonlinear media. Following previous reports on TM SPs of the similar metallic structures, ${ }^{30-32}$ the exact dispersion relations have been obtained analytically without detailed knowledge of the field distributions in the nonlinear media. Moreover, the characteristics of highly propagating terahertz SPs supported by these structures are also investigated. It was shown that, similar to a single layer graphene on Kerr-type nonlinear substrate ${ }^{36}$ if self-focusing nonlinear materials are chosen as the surrounding media of the graphene PPWG, the SPs localization length decreases, while their propagation length remains the same. However, when an appropriate defocusing material is chosen as a substrate in the asymmetric structure in which the cladding is a linear material, optimized SPs can be supported for frequencies larger than $4 \mathrm{THz}$ (The optimized SPs have considerable larger PL and smaller LL than those of a typical graphene PPWG). These results can be useful for modeling high performance plasmonic devices based on graphene PPWG structures. Finally, in order to go beyond the limitation of the values for the field strength and frequency used in the present study, it will be worthwhile for a future effort to re-analyze this nonlinear waveguide problem accounting for the nonlinear optical conductivity of graphene. $^{38}$

${ }^{1}$ K. S. Novoselov, A. K. Geim, S. V. Morozov, D. Jiang, Y. Zhamg, S. V. Dubonos, I. V. Grigarieva, and A. A. Firsov, Science 306, 666 (2004).

${ }^{2}$ K. S. Novoselov, A. K. Geim, S. V. Morozov, D. Jiang, M. I. Katanelson, I. V. Grigorieva, and S. V. Dubones, Nature (London) 438, 197 (2005).

${ }^{3}$ Y. Zhang, J. P. Small, W. V. Pontius, and P. Kim, Appl. Phys. Lett. 86, 073104 (2005).

4Y. Zhang, Y. W. Tan, H. L. Stermer, and P. Kim, Nature 438, 201 (2005).

${ }^{5}$ K. S. Novoselov, Z. Jiang, Y. Zhang, S. V. Morozov, H. L. Stormer, U. Zeitler, J. C. Maan, G. S. Boebinger, P. Kim, and A. K. Geim, Science 315,1379 (2007).

${ }^{6}$ A. H. Castro Neto, F. Guinea, N. M. R. Peres, K. S. Novoseloy, and A. K. Geim, Rev. Mod. Phys. 81, 109 (2009).

${ }^{7}$ F, Bonaccorso, Z, Sun, T. Hasam, and A. C. Ferrari, Nature Fhoton, 4, 611 (2010).

A. Vakil and N. Engheta, Science 332, 1291 (2011).

'F. H. L. Koppens, D. E. Chang, and F. J. Garcia de Abajo, Nano Lett 11, 3370 (2011).

${ }^{10}$ A. N. Grigarenko, M. Polini, and K. S. Novoselov, Nature Photon 6, 749 (2012).

${ }^{11}$ M. Tamagnone, J. S. Gomez-Diaz, J. R. Mosig, and J. Perruisseau-Carrier, J. Appl. Phys. Lott. 101, 214102 (2012).

${ }^{12}$ F. J. Garcia de Abajo, Science 339, 917 (2013).

${ }^{13}$ S. A. Mikhoilov and K. Ziegler, Phys. Rev. Lett. 99, 016803 (2007). 
${ }^{14} \mathrm{G}$. W. Hanson, J. Appl. Phys. 103, 064302 (2008).

${ }^{15}$ G. W. Hanson, A. B. Yakovlev, and A. Mafi, J. Appl. Phys. 110, 114305 (2011).

${ }^{16} \mathrm{M}$. Jablan, H. Buljan, and M. Soljacic, Opt. Express 19, 11236 (2011).

${ }^{17} \mathrm{G}$. W. Hanson, J. Appl. Phys. 104, 084314 (2008).

${ }^{18}$ M. Jablan, H. Buljan, and M. Soljacic, Phys. Rev. B 80, 245435 (2009).

${ }^{19}$ C. H. Gan, H. S. Chu, and E. P. Li, Phys. Rev. B 85, 125431 (2012).

${ }^{20}$ D. Svintsov, V. Vyurkov, V. Ryzhii, and T. Otsuji, J. Appl. Phys. 113, 053701 (2013).

${ }^{21}$ P. I. Buslaev, I. V. Iorsh, I. V. Shadrivov, P. A. Belov, and Y. S. Kivshar, JETP Lett. 97, 535 (2013).

${ }^{22}$ H. Hajian, A. Soltani-Vala, and M. Kalafi, J. Appl. Phys. 114, 033102 (2013).

${ }^{23} \mathrm{H}$. Raether, Surface Plasmons on Smooth and Rough Surfaces and on Grating (Springer, Berlin, 1988).

${ }^{24}$ S. A. Maier, Plasmonics: Fundamentals and Applications (Springer, Berlin, 2007).

${ }^{25}$ Plasmonic Nanoguide and Circuits, edited by S. I. Bozhevolnyi (Pan Stanford, 2009).

${ }^{26}$ V. M. Agranovich, V. S. Babichenko, and V. Y. Chernyak, JETP Lett. 32, 512 (1980). Available at: http:/www.jetpletters.ac.ro/ps/1431/article_21789.shtml.

${ }^{27}$ G. I. Stegeman, C. T. Seaton, J. Ariyasu, R. F. Wallis, and A. A. Maradudin, J. Appl. Phys. 58, 2453 (1985).

${ }^{28}$ J. Ariyasu, C. T. Seaton, G. I. Stegeman, A. A. Maradudin, and R. F. Wallis, J. Appl. Phys. 58, 2460 (1985).

${ }^{29}$ A. D. Boardman, A. A. Maradudin, G. I. Stegeman, T. Twardowski, and B. M. Wright, Phys. Rev. A 35, 1159 (1987).
${ }^{30}$ D. Mihalache, G. I. Stegeman, C. T. Seaton, E. M. Wright, R. Zanoni, A. D. Boardman, and T. Twardowski, Opt. Lett. 12, 187 (1987).

${ }^{31}$ Q. Chen and Z. H. Wang, Opt. Lett. 18, 260 (1993).

${ }^{32}$ J. H. Huang, R. Chang, P. T. Leung, and D. P. Tsai, Opt. Commun. 282, 1412 (2009).

${ }^{33}$ A. R. Davoyan, I. V. Shadrivov, and Y. S. Kivshar, Opt. Express 16, 21209 (2008).

${ }^{34}$ A. R. Davoyan, I. V. Shadrivov, and Y. S. Kivshar, Opt. Express 17, 21732 (2009).

${ }^{35}$ A. R. Davoyan, I. V. Shadrivov, and Y. S. Kivshar, Opt. Lett. 36, 930 (2011).

36. Wang, W. Cai, X. Zhang, and J. Xu, Opt. Lett. 37, 2730 (2012).

${ }^{37}$ A. Y. Nikitin, F. Guinea, F. J. Garcia-Vidal, and L. Martin-Moreno, Phys. Rev. B 84, 195446 (2011).

${ }^{38}$ D. A. Smimova, A. V. Gorbach, I. V. Iorsh, I. V. Shadrivov, and Y. S. Kivshar, Phys. Rev. B 88, 045443 (2013).

${ }^{39}$ M. Liu, X. Yin, and X. Zhang, Nano Lett. 12, 1482 (2012).

${ }^{40}$ V. Ryzhii, T. Otsuji, M. Ryzhii, V. G. Leiman, S. O. Yurchenko, V. Mitin, and M. S. Shur, J. Appl. Phys. 112, 104507 (2012).

${ }^{41}$ L. A. Falkovsky and S. S. Pershoguba, Phys. Rev. B 76, 153410 (2007).

${ }^{42}$ L. A. Falkovsky, J. Phys.: Conf. Ser. 129, 012004 (2008).

${ }^{43}$ H. Hajian, A. Soltani-Vala, and M. Kalafi, Opt. Commun. 292, 149 (2013).

${ }^{44}$ S. A. Mikhailov, Europhys. Lett. 79, 27002 (2007).

${ }^{45}$ E. Hendry, P. J. Hale, J. Moger, A. K. Savchenko, and S. A. Mikhailov, Phys. Rev. Lett. 105, 097401 (2010). 